\title{
Band gap and double-negative properties of a star-structured sonic metamaterial
}

\author{
Meng Chen ${ }^{\mathrm{a}, \mathrm{b}}$, Wenshuai Xu ${ }^{\mathrm{a}, \mathrm{b}}$, Yu Liu ${ }^{\mathrm{a}, \mathrm{b}}$, Kuo Yan ${ }^{\mathrm{c}}$, Heng Jiang ${ }^{\mathrm{a}, \mathrm{b}, *}$, Yuren Wang ${ }^{\mathrm{a}, \mathrm{b}, *}$ \\ ${ }^{a}$ Key Laboratory of Microgravity, Institute of Mechanics, Chinese Academy of Sciences, Beijing 100190, People's Republic of China \\ ${ }^{\mathrm{b}}$ University of Chinese Academy of Sciences, Beijing 100049, People's Republic of China \\ ${ }^{\mathrm{c}}$ College of Materials Science and Chemical Engineering, Harbin Engineering University, Harbin 150001, People's Republic of China
}

\section{A R T I C L E I N F O}

\section{Keywords:}

Sonic metamaterials

Effective medium parameter

Double-negative

Single-phase

Star-shaped structure

\begin{abstract}
A B S T R A C T
Sonic metamaterials have a wide range of applications in wave control and super-resolution imaging, and are favored for their several unique and advantageous properties. However, current double-negative sonic metamaterials have complex structures composed of various materials, which limits their design and application. Thus, we must produce double-negative features using a simple structure of one material. Because of their unique concave configurations and various resonances, star-shaped structures readily form band gaps and show superior material properties. In this study, we designed and simulated star-shaped single-phase metamaterials, considered ideal structures. Our numerical results suggest that these metamaterials have two band gaps as well as double-negative properties over specific frequency ranges. Moreover, we investigated how their band gap and double-negative properties depended on the concave angle.
\end{abstract}

\section{Introduction}

Sonic metamaterials [1] are artificial composite materials with acoustic characteristics not found in natural materials, such as negative effective mass density [2-4] and negative effective modulus [5,6]. Negative effective mass density means that the direction of acceleration and driving force of the medium are opposite under dynamic pressure; a negative effective modulus can be interpreted as a scattering field generated by a resonance that is much larger than the incident field, producing contrary changes in pressure and volume. Having both negative effective modulus and density, sonic metamaterials are compelling because of their negative refraction over a certain frequency range [7] as well as the inverse Doppler effect [8] and super-resolution imaging [9-14].

Generally, sonic metamaterials gain double-negative properties by being composed of materials with different elastic constants, which forms hybrid resonances by accessing the various resonance modes of the structural units [15]. Thus, double-negative acoustic metamaterials often have complex structures and must be composed of multiple materials. However, such structures are difficult to design and use. Thus, it is important to study how to produce double-negative properties using a single-phase material in a simple structure in order to better design and develop acoustic devices made of metamaterials.

Using single-phase materials to achieve the double-negative property is challenging because this extraordinary property depends on the vibration characteristics of the artificial "atomic" (the sub-wavelength locally resonant units) in a specific frequency range. Producing double-negativity requires introducing a resonant unit with both dipole and monopolar resonances in its structure [15-20]. Negative mass is usually achieved by introducing a lumped mass into the sub-wavelength structure constituting the spring resonant unit [16]; the units show negative effective density at the dipole resonance frequency. To produce a negative effective modulus, a Helmholtz resonator or a rotating resonator $[5,21]$ is usually introduced into the sub-wavelength structure, producing a negative effective modulus near the monopolar resonance frequency. Hybridization states and chiral structures are also used in designing double-negative acoustic metamaterials [22,23].

Based on the above considerations, Liu et al. produced double-negative metamaterials by introducing a chiral structure, which had a negative group velocity over specific frequency ranges [22,23]. Using four resonant units, Lai et al. formed a hybridized elastomeric solid and produced a two-dimensional double-negative metamaterial based on hybrid resonance states [15]. Yang et al. achieved double-negative properties in a double-layer film system [19]; if the double membranes vibrate in phase, the dipole vibration provides a negative effective mass density, whereas if the double membranes vibrate in opposite phase, the monopole vibration provides a negative effective modulus. However, these double-negative metamaterials have multiple material

\footnotetext{
* Corresponding authors at: Key Laboratory of Microgravity, Institute of Mechanics, Chinese Academy of Sciences, Beijing 100190, People's Republic of China.

E-mail addresses: hengjiang@imech.ac.cn (H. Jiang), yurenwang@imech.ac.cn (Y. Wang).
} 
phases, and their complex structures are difficult to use. Although Zhu et al. developed a single-phase metamaterial with a chiral microstructure-which can achieve both negative effective mass density and modulus owing to simultaneous translational and rotational resonances-the structure is also complicated and bulky. Thus, it is necessary to introduce new resonance mechanisms containing both dipole and monopole resonances in a lightweight structure if single-phase metamaterials are to be developed.

Materials with star-shaped structures possess a negative Poisson's ratio from its special concave configuration. Compared with traditional materials, the star-shaped structure has higher energy absorption efficiency, better designability, homodromous bending capability, and low mass [24-26]. Its mechanical properties can be adjusted by adjusting its geometry, such as its thickness, beam length, and beam concavity. Considering their wave characteristics, with volume changes and various resonance behaviors, the star-shaped structure is good at forming a low-frequency band gap and extraordinary properties because of its concave configuration. In this paper, we systematically study the formation mechanism of band gaps in star-shaped sonic metamaterials. Revealed by numerical analysis, this star-shaped structure shows special resonance properties, achieves wide band gaps, and has the doublenegative property in a specific frequency range. We also investigate how its geometry affects its band gap, as described below.

\section{Star-shaped structure model and numerical calculation method}

Depending on its cell structure, star-shaped structures are typical auxetics that come in either a four-pointed or six-pointed star $[25,26]$. Its structure is made of slender beams, so it is very light and enables a variety of resonances. For example, the presence of a lumped mass in the concave node produces a dipole resonance, while bending of the cant beams and rotation of the point mass induces a monopolar resonance. These resonances enable the superior acoustic properties such as negative effective density and negative effective modulus. In this paper, we focus on the wave characteristics of the four-pointed star, the unit cell of which is given in Fig. 1.

Although the thickness of the structure will affect the position of its band gap, we only studied two-dimensional structures in this paper [27]. This structure consists of four straight beams of equal length $L_{1}$ and eight star-shaped cant beams of equal length $L_{2}$. The beams have

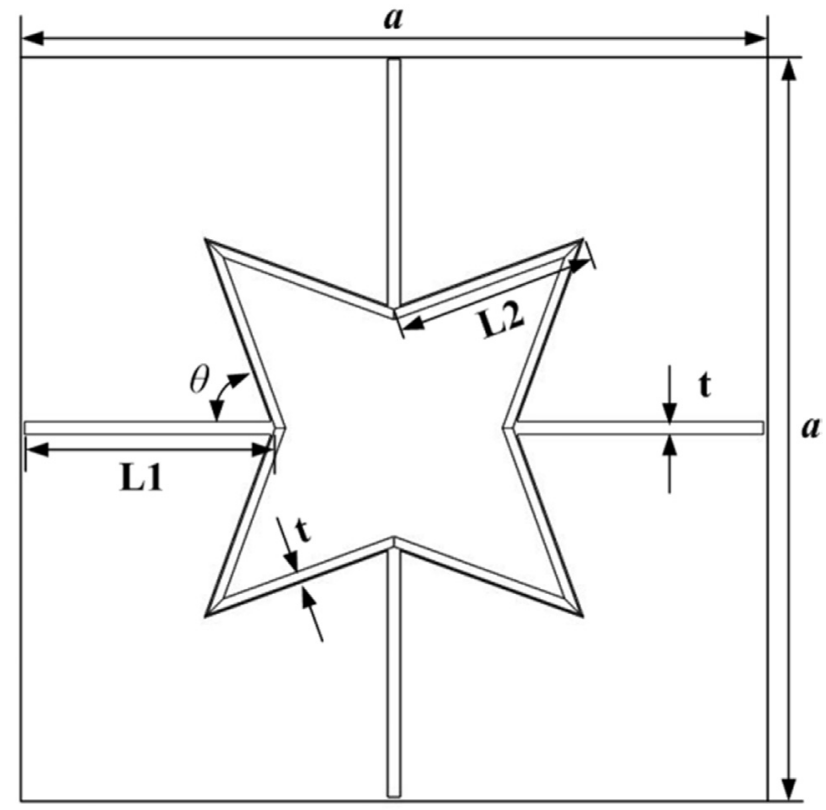

Fig. 1. Schematic of the unit four-pointed star structure. equal thickness $t$. The counterclockwise angle between adjacent cell walls is denoted as $\theta$. The unit cells are arranged in a square lattice with the lattice constant $a$, which can be expressed as $2\left\{\frac{\sin \left(\theta-45^{\circ}\right)}{\sin \left(45^{\circ}\right)} \cdot L_{2}+L_{1}\right\}$. In the calculations, we set $L_{1}, L_{2}$, and $t$ to $0.5 \mathrm{~cm}, 1 \mathrm{~cm}$, and $0.05 \mathrm{~cm}$, respectively. When $L_{1}, L_{2}$, and $t$ are constant, the angle $\theta$ of the starshaped structure must be more than $45^{\circ}$ and less than $135^{\circ}$. The whole structure is given the material properties of steel.

The band structure, vibration modes, and effective medium parameters of the star-shaped structure were all studied using COMSOL Multiphysics finite-element method (FEM) software. For two-dimensional sonic metamaterials, the displacement can be divided into an in$x, y$ plane and an out- $x, y$ plane. We only considered the fluctuations in the $x-y$ plane in this study. In the calculations, periodic boundary conditions are imposed to simplify the computational domain to one unit cell.

$u(x, y+a)=u(x, y) e^{i\left(k_{y} \cdot a\right)}$,

$v(x, y+a)=v(x, y) e^{i\left(k_{y} \cdot a\right)}$

Based on Bloch's theorem, solving the $k-w$ relations on the boundary of the Brillouin zone yields the dispersion relation for the whole structure $[28,29]$. When calculating transmission loss, the displacements of the finite elements are taken in one direction only; the periodic boundary conditions account for an infinite array of units in the other direction. Assuming that the incident plane wave is on the surface of the structure, we determine the transmission loss of the starshaped structure from the transmission coefficient. Meanwhile, to reduce the reflected wave at the boundary, a perfect matching layer is used in the incident direction. Under the long-wave hypothesis, the acoustic metamaterials can be regarded as homogeneous, with special properties according to the equivalent medium theory. Also, the dynamic behavior can be represented using equivalent medium parameters to determine the propagation of the acoustic wave. The effective parameters of the medium were calculated by determining the displacement, strain, stress, and force on the boundaries; the details of our calculation methods can be found elsewhere $[15,30]$.

The effective mass was calculated according to Newton's second law:

$\rho^{e f f}=\frac{m^{e f f}}{a^{2}}=\frac{F_{x}^{e f f}}{\ddot{u}_{x}^{e f f} a^{2}}=\frac{=-F_{x}^{e f f}}{\omega^{2} u_{x}^{e f f} a^{2}}$

where $C_{11}^{\text {eff }}, C_{12}^{e f f}$, and $C_{44}^{e f f}$ are the effective stiffness tensors; $T_{x x}^{\text {eff }}, T_{y y}^{\text {eff }}$, and $T_{x y}^{e f f}$ are the xx, yy, and xy components of effective stress tensor. $S_{x x}^{e f f}, S_{y y}^{e f f}$, and $S_{x y}^{e f f}$ are $\mathrm{xx}, \mathrm{yy}$, and xy components of the effective strain tensor. Under external stimulation, there are three unknowns in the constitutive relations: $C_{11}^{\text {eff }}, C_{12}^{\text {eff }}$, and $C_{44}^{\text {eff }}$. The others can be obtained from the stress and deformations of unit boundary.

$T_{x x}^{e f f}, T_{y y}^{e f f}$, and $T_{x y}^{e f f}$ were calculated as follows:

$T_{x x}^{e f f}=\frac{\left.\int T_{x x} d y\right|_{x=0}+\left.\int T_{x x} d y\right|_{x=a}}{2 a}$

$T_{y y}^{e f f}=\frac{\left.\int T_{y y} d x\right|_{y=0}+\left.\int T_{y y} d x\right|_{y=a}}{2 a}$

$T_{x y}^{e f f}=\frac{\left.\int T_{x y} d x\right|_{y=0}+\left.\int T_{x y} d x\right|_{y=a}}{2 a}$

$S_{x x}^{e f f}, S_{y y}^{e f f}$, and $S_{x y}^{e f f}$ were calculated as follows:

$S_{x x}^{e f f}=\frac{\left.\int u_{x} d y\right|_{x=a}-\left.\int u_{x} d y\right|_{x=0}}{a^{2}}$

$S_{y y}^{e f f}=\frac{\left.\int u_{y} d y\right|_{x=a}-\left.\int u_{y} d y\right|_{x=0}}{a^{2}}$

$S_{x y}^{e f f}=\frac{\left.\int u_{x} d x\right|_{y=a}-\left.\int u_{x} d x\right|_{y=0}+\left.\int u_{y} d y\right|_{x=a}-\left.\int u_{y} d y\right|_{x=0}}{2 a^{2}}$

The effective bulk modulus $\kappa^{\text {eff }}$ and effective shear modulus $\mu^{\text {eff }}$ were defined by $C_{11}^{\text {eff }}, C_{12}^{\text {eff }}$, and $C_{44}^{\text {eff }}$ : 


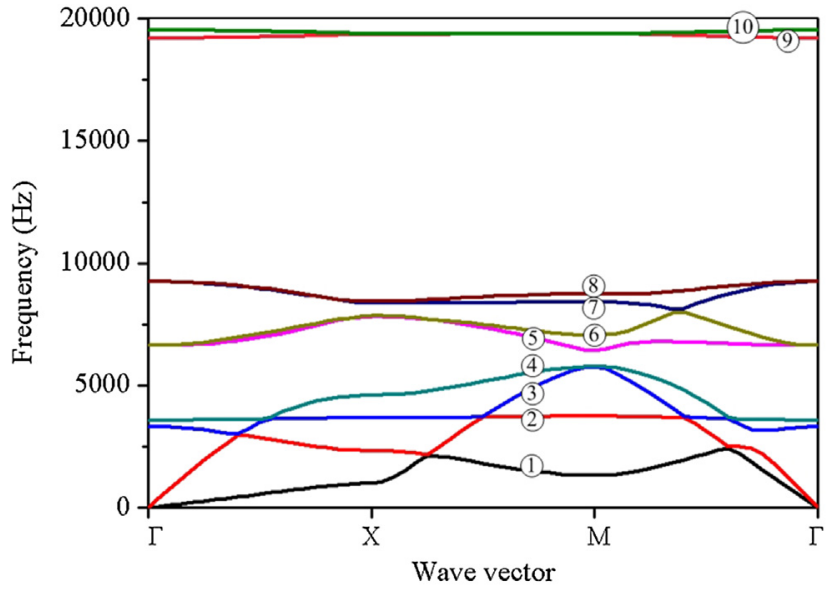

Fig. 2. Band structure of the star-shaped auxetic structure $\left(\theta=60^{\circ}\right)$.

$\kappa^{e f f}=\frac{c_{11}^{e f f}+c_{12}^{e f f}}{2}$

$\mu^{e f f}=\frac{c_{11}^{e f f}-c_{12}^{e f f}}{2}$

\section{Results and discussion}

\subsection{Band structure and vibration modes of the star-shaped structure}

Using the above methods, we studied the band structure, vibration modes, and effective medium parameters of the star-shaped structure. Fig. 2 shows the band structure of the $60^{\circ}$ star-shaped structure. Two wide band gaps exist within the calculated frequency range; the first band gap appears between the fourth and fifth branches from $5780 \mathrm{~Hz}$ to $6433.7 \mathrm{~Hz}$ with a bandwidth of $653.7 \mathrm{~Hz}$; the second band gap exists between the eighth and ninth branches from $9253.8 \mathrm{~Hz}$ to $19,204 \mathrm{~Hz}$ with a bandwidth of $9950.2 \mathrm{~Hz}$. Based on the center frequencies of the first and second band gaps, the longitudinal wavelengths are $0.85 \mathrm{~m}$ and $0.36 \mathrm{~m}$, respectively. At only $0.025 \mathrm{~m}$, the lattice constant of the star-shaped auxetic structure is smaller than the wavelength of either band gap. Thus, the band gaps of the star-shaped structure arise from local resonances. Because of Fano-like interference (the response spectrum has an antisymmetric resonance peak), the traditional locally resonant sonic metamaterials have narrow bandwidth [31]. Moreover, the second band width of the star-shaped structure $(9950 \mathrm{~Hz})$ is much wider than that of traditional locally resonant sonic metamaterials. The star-shaped structure shows behavior like low-frequency wave filtering and wide-frequency vibration isolation.

We also investigated the vibration modes at the cutoff frequency (Fig. 3); in this figure, the black (colored) lines mark the original (deformed) shape of the unit cell. The vibration mode corresponding to the lower edge of the first band gap entail the bending of two straight beams and cant beams, while another two straight beams remain still [Fig. 3(a)]. This result is similar to the vibration mode at the lower-edge frequency of a three-component locally resonant photonic crystal (LRPC) [2]. The vibration mode at the upper edge of the first band gap [Fig. 3(b)] entails an outward motion of the four straight beams, causing bending deformation of the cant beams. The adjacent cell then becomes larger at the star-shaped connection point. The whole cell maintains a dynamic balance similar to the monopole vibration mode and thus can be regarded as a rotation of the points of the star.

The vibration mode at the lower edge of the second band gap [Fig. 3(c)] involves two straight beams bending while the other two straight beams move in the same direction along the extension line of the beams. Although the cant beams emerge bent, the center of mass of the cant beams moves relative to that of the straight beams. Hence this vibration mode is similar to the vibration mode of a dipole. The vibration mode at the upper edge of the second band gap [Fig. 3(d)] entails the four straight beams bending considerably while the cant beam deform less.

These vibration modes of the star-shaped structure are quite different from traditional LRPCs. At the cutoff frequency, their deformations mainly feature bending, which for beams allows monopoles, dipoles, and rotational resonances to form easily with concomitant band gaps.

\subsection{Equivalent medium parameters of the star-shaped structure and formation mechanism of double-negative properties}

The slope of the eighth dispersion curve of the star-shaped structure (Fig. 2) is negative along the $\Gamma X$ direction, implying a negative group velocity in this frequency range. According to the equivalent medium theory of acoustic metamaterials [15], negative group velocity means negative refraction; that is, both the equivalent mass density and equivalent modulus of the star-shaped structure in this range are negative. To further confirm the double-negative properties, we calculated the equivalent medium parameters for the star-shaped structure with $L_{1}=0.5 \mathrm{~cm}, L_{2}=1 \mathrm{~cm}$, and $\theta=60^{\circ}$. With a lattice constant of about $2.5 \mathrm{~cm}$, the wavelength is about six times that of the lattice constant in the frequency band of $8400-9400 \mathrm{~Hz}$, and the wave characteristics can be described by effective parameters. From the dispersion curve, the equivalent mass density, the equivalent bulk modulus, and the equivalent shear modulus of the eighth curve along the $\Gamma \mathrm{X}$ direction [Fig. 4(a)-(d)], the dispersion-curve frequencies between $8400 \mathrm{~Hz}$ and $9400 \mathrm{~Hz}$ show anomalous behavior: they show negative slopes, and all three equivalent medium parameters of the eighth curve are negative, corresponding to double-negative properties.

To study the formation mechanism of the double-negative characteristics, we generated a vibration mode at the midpoint of the $\Gamma \mathrm{X}$ direction of the eighth dispersion curve (Fig. 5). This mode mainly involves beam bending, which can be decomposed into translational motion along the horizontal straight beams and bending of the other beams. The translational motion of the horizontally straight beams is similar to the vibration mode of an LRPC at the lower edge of the band gap, as for a dipole vibration [4], which can produce negative effective mass density. Meanwhile, the bending of the other beams is equivalent to rotational deformation of the four points of the star, which provides the negative effective modulus. We find that the double-negative properties of the star-shaped structures can be produced by a hybrid state that consists of a dipole resonance and a rotating state arising from bending. In contrast to traditional hybrid double-negative acoustic metamaterials, the star-shaped structure is simpler, being made from just a single-phase material. Moreover, the six-pointed star structure will have similar wave characteristics due to its similar structural features. However, the frequency position of the band gap and the negative parameter will be very different because the structure has different symmetry.

Based on effective medium theory, the longitudinal wave velocity is an imaginary number if either $\kappa_{\text {eff }}+\mu_{\text {eff }}$ or $\rho_{\text {eff }}$ is negative, creating a wave that attenuates exponentially but cannot propagate through the structure. Equally, when either $\mu_{\text {eff }}$ or $\rho_{\text {eff }}$ is negative, a transverse wave attenuates rapidly but also cannot propagate. For the star-shaped structure, all three parameters- $\kappa_{\text {eff, }}, \mu_{\text {eff, }}$, and $\rho_{\text {eff }}$-are negative in the eighth dispersion curve, and both longitudinal and transverse waves propagate with negative refraction. In the frequency range of 9400-19,204 Hz, $\rho_{\text {eff }}$ is positive but $\kappa_{\text {eff }}$ and $\mu_{\text {eff }}$ are negative because of the rotating resonators. Moreover, the longitudinal and transverse waves cannot propagate, forming the band gap.

To further verify these results, we used FEM to calculate the transmission characteristics of the star-shaped structure (Fig. 6). In this simulation, four finite units were configured in the horizontal direction. 


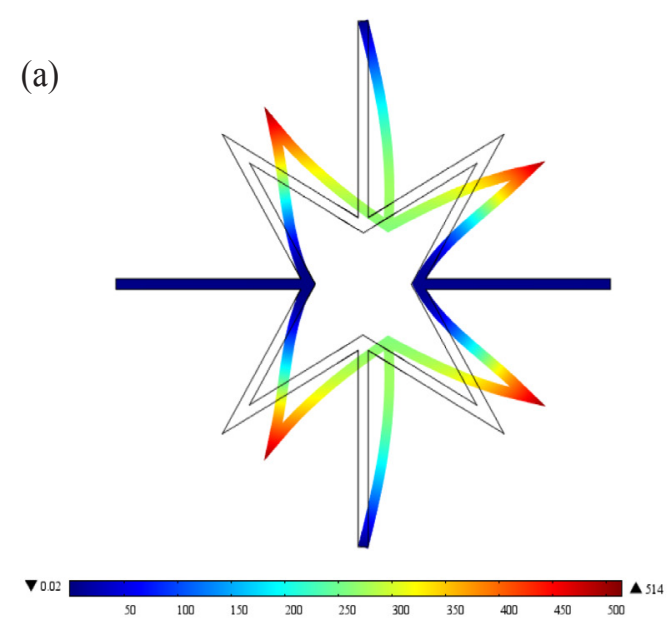

$5780 \mathrm{~Hz}$

(c)

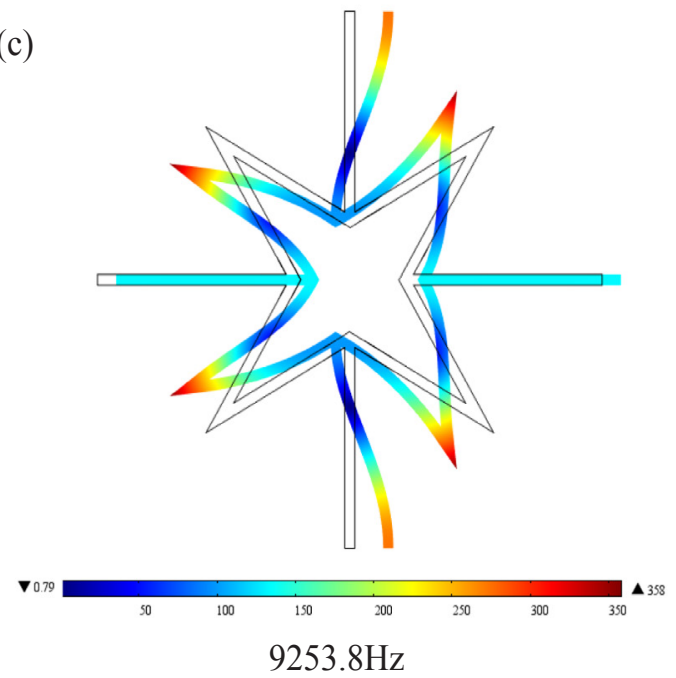

(b)

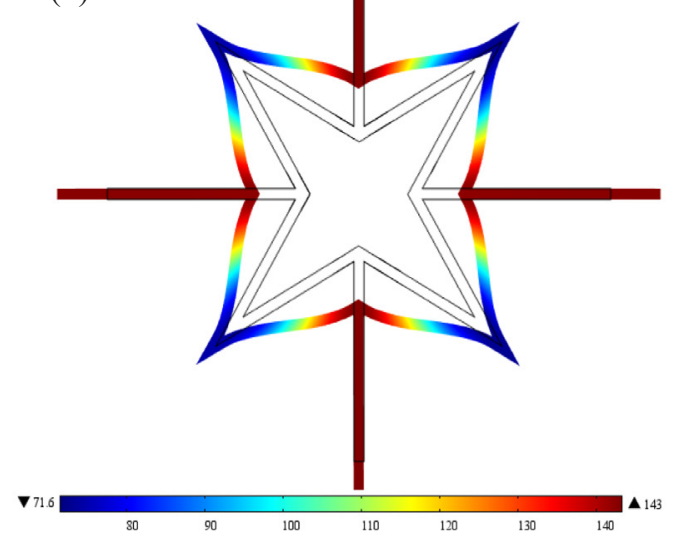

$6433.7 \mathrm{~Hz}$

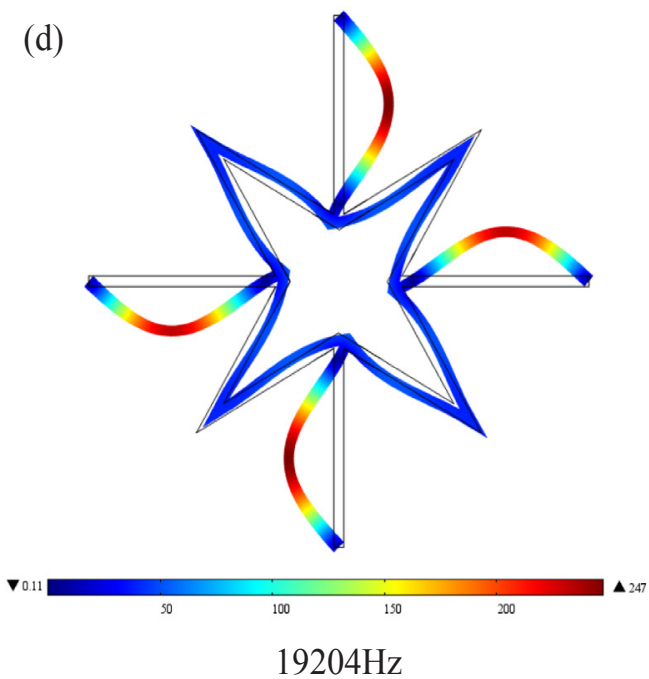

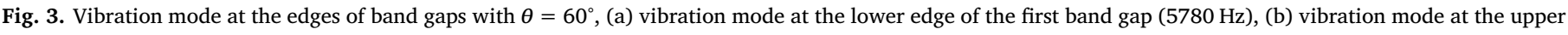

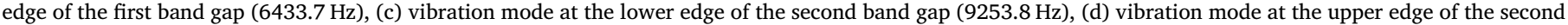
band gap $(19,204 \mathrm{~Hz})$.

As shown in the transmission characteristic curve of the star-shaped structure [Fig. 6(b)] for frequencies up to $20,000 \mathrm{~Hz}$ along the $\Gamma \mathrm{X}$ direction, significant vibration attenuation appears in the frequency ranges of $4750-6500 \mathrm{~Hz}$ and $9500-19,200 \mathrm{~Hz}$. For acoustic metamaterials, when either effective mass density or effective modulus is negative, the wave velocity is an imaginary number, and the wave will decay rapidly in the structure and then form the band gaps. In the frequency range of the double-negative parameters, the wave can propagate [15]. A band gap forms in the two frequency ranges consistent with the calculated band gap structure and equivalent medium parameters. Note also that the star-shaped structure can provide wideband isolation of an elastic wave at low frequencies, which may be useful in designing new filters and sound insulators.

3.3. Influence of concave angle on band-gap structure and double-negative properties of star-shaped structure

The star-shaped structure has many structural parameters, including the concave angle $\theta$ in the joint, the length of the straight beams $L_{1}$, the length of the cant beam $L_{2}$, and the thickness of beams $t$. Changing these parameters can change the mechanical properties and the shape of the band structure. Previous work has suggested that changing the concave angle $\theta$ changes Poisson's ratio and the effective static elastic modulus. To study how the concave angle affects the characteristics of the starshaped structure, we calculated the band structure of the star-shaped structure for $\theta$ of $50-120^{\circ}$, letting us analyze how it affected the width and position of the band gap. In these calculations, $L_{1}$ and $L_{2}$ are constant. Fig. 7 shows the angular dependence of the cutoff frequency for the first and second band gaps.

We find that only star-shaped structures with concave angles of $50-70^{\circ}$ generate a first band gap (Fig. 7). At $70^{\circ}$, the band gap widens slightly for the upper edge frequencies. Recalling Fig. 2, the first band gap tends to appear between the fourth and fifth dispersion curves, and the cutoff frequency is at the $\mathrm{M}$ point. To further reveal the formation mechanism of the first band gap, we calculated the vibration modes at the $\mathrm{M}$ point of the fourth and fifth dispersion curves at different concave angles (Fig. 8).

For the fourth dispersion curve, the vibration modes at different 

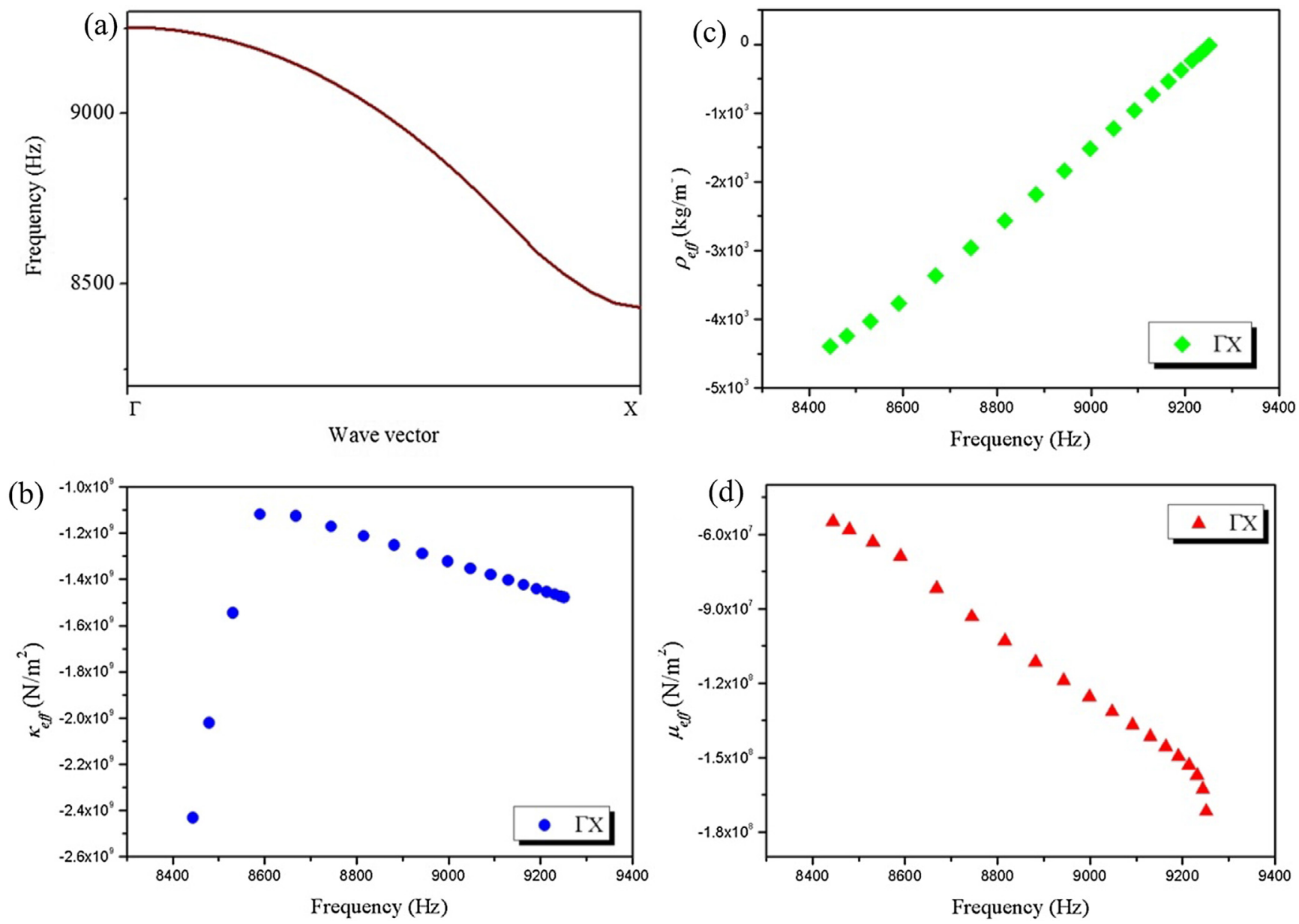

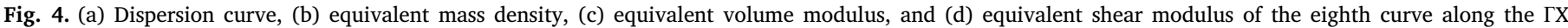
direction.

angles are similar; specifically, the opposite straight beams and cant beams bend while the other two connecting beams stay fixed. In contrast, for the $\mathrm{M}$ point of the fifth dispersion curve, the vibration modes are quite different. If the concave angle is $80^{\circ}$ or more, the vibration modes are similar to those of the fourth dispersion curve, but with a different change in the direction of vibration. Hence, the first band gap disappears with these opposing modes from the fourth and fifth dispersion curves. If the concave angle is $50-70^{\circ}$, the vibration mode entails outward deformation or bending of the four straight beams, accompanied by bending of the by the cant beams. The whole structure then shows a rotational resonance state that produces a large difference in the vibration mode at the $\mathrm{M}$ point of the fourth dispersion curve. Hence the band gap appears because the degenerate state is suppressed. The first band gap is correlated with that at the M point of the fifth
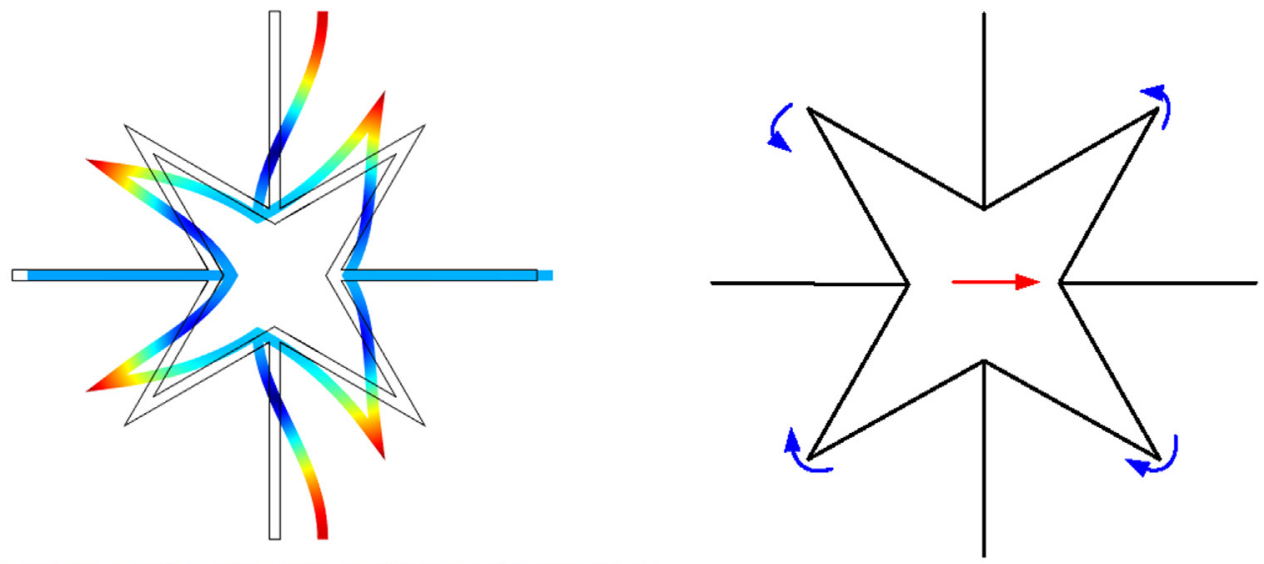

$\mathbf{\nabla} 0.47$

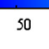

100

150

200

250

300

Fig. 5. (a) Vibration mode and (b) simplified vibration at the midpoint of the eighth band slope in the $\Gamma X$ direction. 


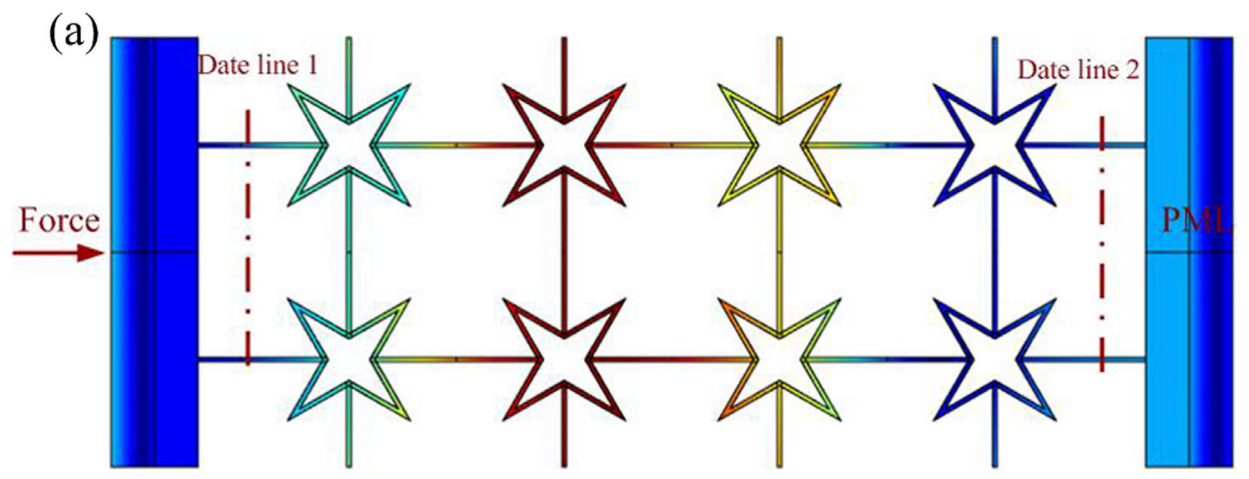

(b)

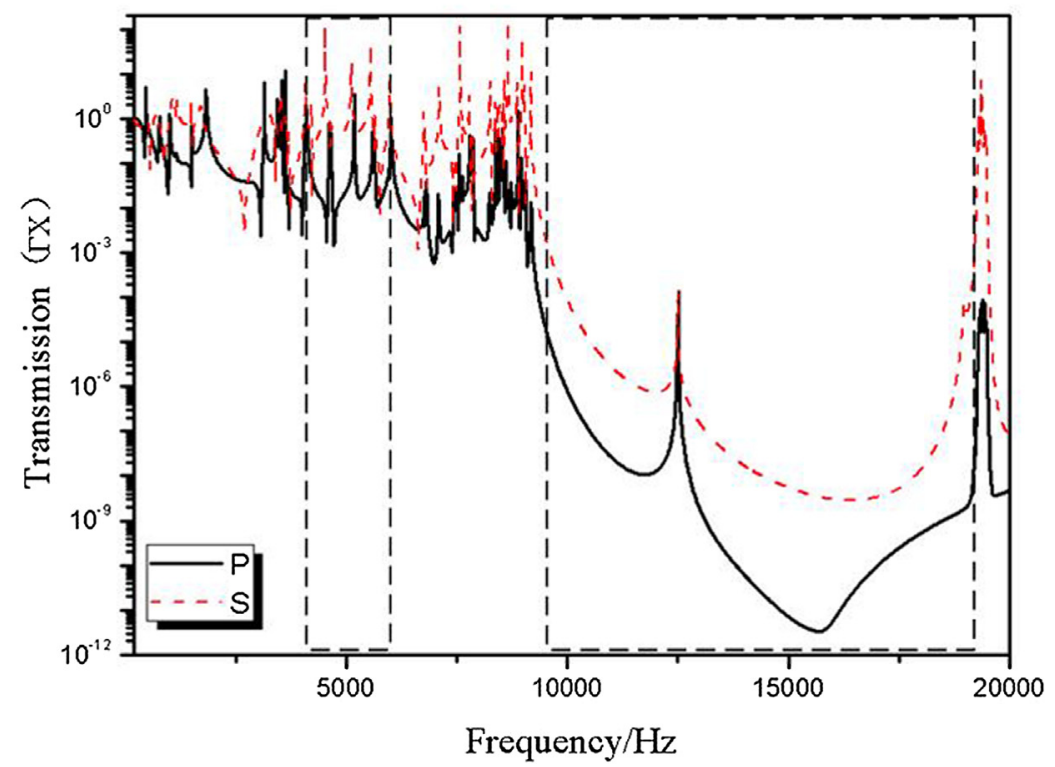

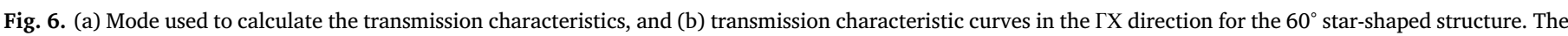
transmissions for the longitudinal and transverse waves are denoted as $p$ and $s$, respectively.

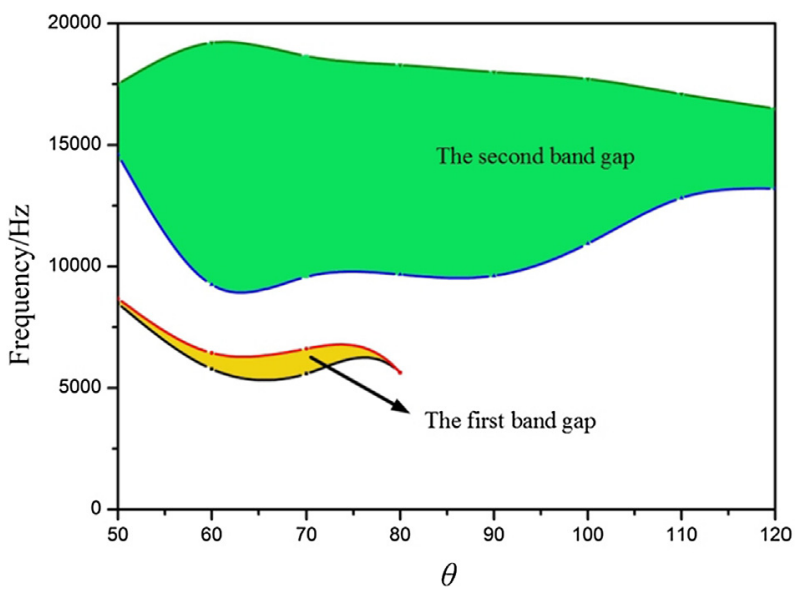

Fig. 7. Dependence of the cutoff frequency for the band gaps on concave angle.

dispersion curve. At angles of $50-70^{\circ}$, the fourth and the fifth dispersion curves separate because of the bending rotational resonance state at the $\mathrm{M}$ point, producing a low-frequency band gap.

As shown by the dependence on angle of the cutoff frequency for the second band gap (Fig. 7), the lower-edge frequencies decrease initially and then increase gradually, while the upper-edge frequencies increase initially and then decrease gradually. The maximum band gap width is at $60^{\circ}$. For the second band gap, the width of the band gap mainly depends on the position of the $\mathrm{M}$ point of the eighth band curve. At a concave angle of $50^{\circ}$, the $\mathrm{M}$ point of the eighth dispersion curve is located above the $\Gamma$ point, producing a narrow second band gap. Above $50^{\circ}$, the $\mathrm{M}$ point of the eighth dispersion curve is located below the $\Gamma$ point, producing a wide band gap.

As shown by the vibration modes at the M point of the eighth band curve (Fig. 9), the unit cell deformations are similar at various concave angles. Four straight beams move outward, opening the concave angle. For concave angles of $60^{\circ}, 70^{\circ}$, and $80^{\circ}$, the structure mainly bends and undergoes overall rotation. These results show that bending is better for generating a broad band gap at the $M$ point of the eighth band curve at concave angles of $60-80^{\circ}$. The band structure is quite different at various concave angles, revealing that varying the concave angle can effectively control the band structure.

\section{Conclusions}

By using FEM, we investigated the band-gap formation mechanisms and effective parameters of a four-pointed star structure. This auxetic metamaterial can generate two broad band gaps at low frequency, which arise from the abundant resonance modes generated by bending the beams. Calculated parameters verified that the single-phase star- 
(a)
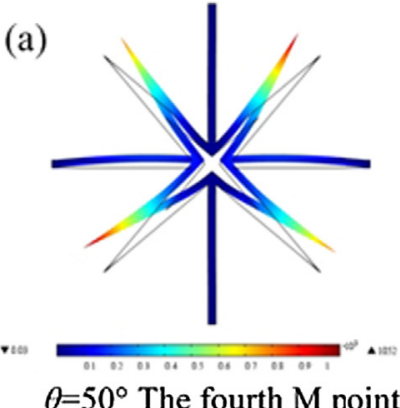

$\theta=50^{\circ}$ The fourth $\mathrm{M}$ point

(c)

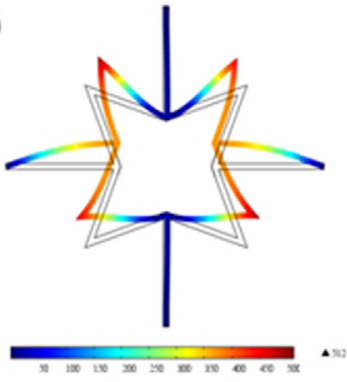

$\theta=70^{\circ}$ The fourth $\mathrm{M}$ point

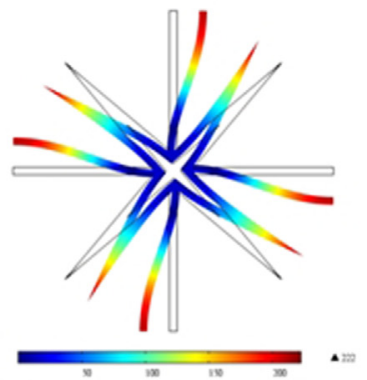

$\theta=50^{\circ}$ The fifth $\mathrm{M}$ point (b)

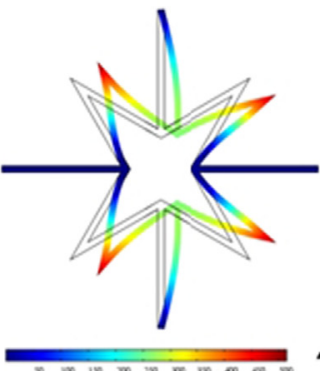

$\theta=60^{\circ}$ The fourth $\mathrm{M}$ point

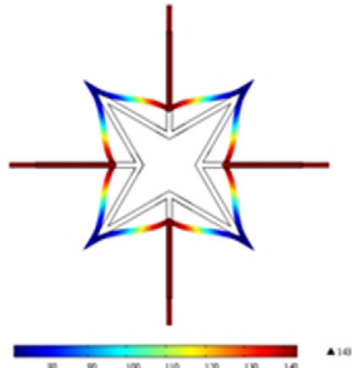

$\theta=60^{\circ}$ The fifth $\mathrm{M}$ point

Fig. 8. Vibration-mode dependence on concave angle at the M point of the fourth and fifth dispersion curves.
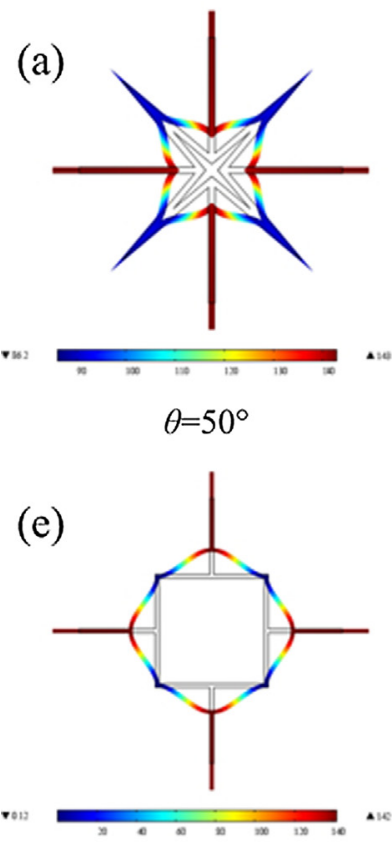

(e)

$\theta=90^{\circ}$

$$
\theta=50^{\circ}
$$

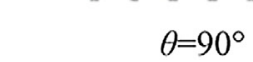

(b)

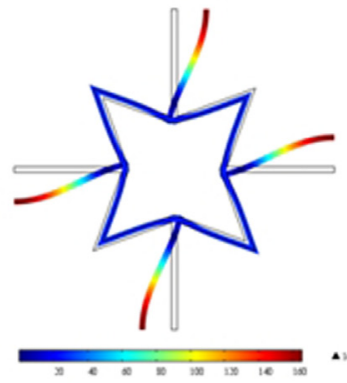

$\theta=70^{\circ}$ The fifth $\mathrm{M}$ point (d)

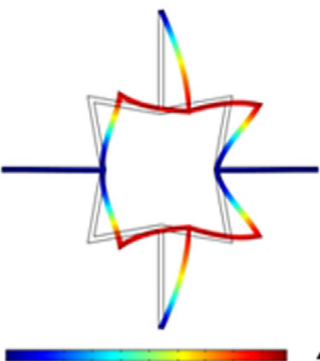

$\theta=80^{\circ}$ The fourth $\mathrm{M}$ point

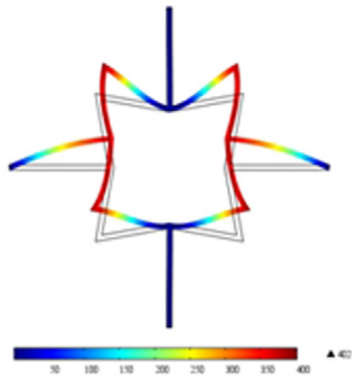

$\theta=80^{\circ}$ The fifth $\mathrm{M}$ point

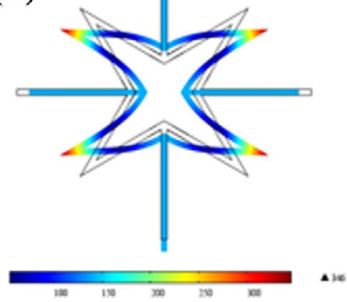

$\theta=60^{\circ}$

(f)

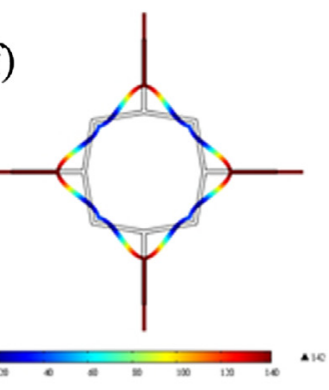

$\theta=100^{\circ}$ (c)

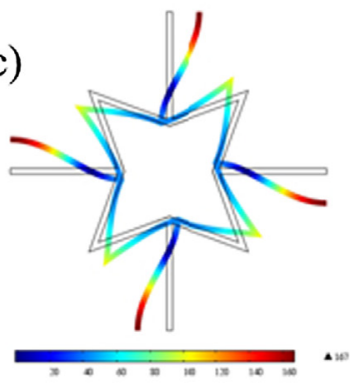

$\theta=70^{\circ}$

(g)

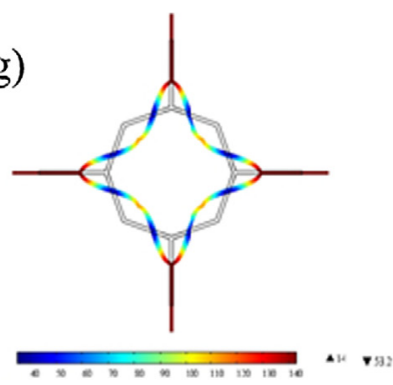

$\theta=110^{\circ}$ (d)

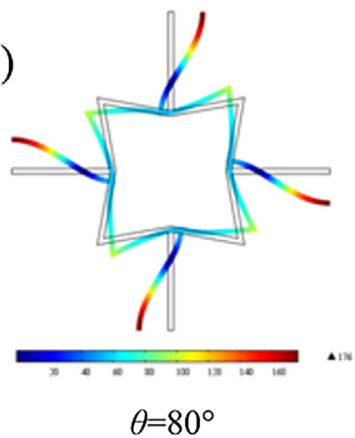

(h)

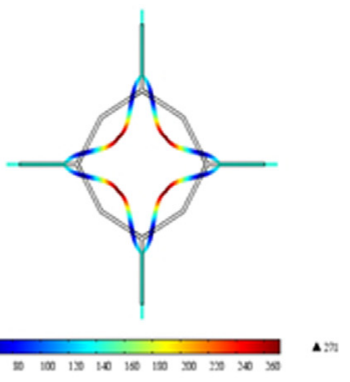

$\theta=120^{\circ}$

Fig. 9. Vibration modes at the M point of the eighth dispersion curve with different concave angles.

shaped structure could achieve double-negative properties owing to the hybrid state formed under bending. Moreover, we could adjust the position and width of the band gap by varying the concave angle and other structural parameters. These results show that the double-negative property and a lower band gap can be produced in a single-phase material in a simple, lightweight structure. This metamaterial provides a new way to produce a lightweight acoustic lens and to achieve subwavelength sound focusing and super-resolution imaging.

\section{Acknowledgments}

We acknowledge support from the National Natural Science Foundation of China (Grant Nos. 11202211 and 11602269), the Strategic Priority Research Program of the Chinese Academy of Sciences (Grant No. XDB22040301), and the Research Program of Beijing (Grant Nos. Z161100002616034 and Z171100000817010). 


\section{References}

[1] Lu MH, Feng L, Chen YF. Phononic crystals and acoustic metamaterials. Mater Today 2009;12(12):34-42.

[2] Liu Z, Zhang X, Mao Y, Zhu YY, Yang Z, Chan CT, et al. Locally resonant sonic materials. Science 2000;289(5485):1734-6.

[3] Yang Z, Mei J, Yang M, Chan NH, Sheng P. Membrane-type acoustic metamaterial with negative dynamic mass. Phys Rev Lett 2008;101(20):204301.

[4] Lee SH, Park CM, Seo YM, Wang ZG, Kim CK. Acoustic metamaterial with negative density. Phys Lett A 2009;373(48):4464-9.

[5] Fang N, Xi D, Xu J, Ambati M, Srituravanich W, Sun C, et al. Ultrasonic metamaterials with negative modulus. Nat Mater 2006;5(6):452-6.

[6] Lee SH, Park CM, Seo YM, Wang ZG, Kim CK. Acoustic metamaterial with negative modulus. J Phys: Condens Mat 2009;21(17):175704.

[7] Pendry JB. A chiral route to negative refraction. Science 2004;306(5700):1353-5.

[8] Seddon N, Bearpark T. Observation of the inverse Doppler effect. Science 2003;302(5650):1537-40.

[9] Grbic A, Eleftheriades GV. Overcoming the diffraction limit with a planar lefthanded transmission-line lens. Phys Rev Lett 2004;92(11):117403.

[10] Zhang S, Yin L, Fang N. Focusing ultrasound with an acoustic metamaterial network. Phys Rev Lett 2009;102:194301.

[11] Ambati M, Fang N, Sun C, Zhang X. Surface resonant states and superlensing in acoustic metamaterials. Phys Rev B 2007;75:195447.

[12] Liu F, Cai F, Peng S, Hao R, Ke M, Liu Z. Parallel acoustic near-field microscope: A steel slab with a periodic array of slits. Phys Rev E 2009;80:026603.

[13] Zhou X, Hu G. Superlensing effect of an anisotropic metamaterial slab with nearzero dynamic mass. Appl Phys Lett 2011;98:263510.

[14] Deng K, Ding Y, He Z, Zhao H, Shi J, Liu Z. Theoretical study of subwavelength imaging by acoustic metamaterial slabs. J Appl Phys 2009;105:124909.

[15] Lai Y, Wu Y, Sheng P, Zhang ZQ. Hybrid elastic solids. Nat Mater 2011;10(8):620-4.

[16] Li J, Chan CT. Double-negative acoustic metamaterial. Phys Rev E 2004;70(5):055602.

[17] Wu Y, Lai Y, Zhang ZQ. Elastic metamaterials with simultaneously negative effective shear modulus and mass density. Phys Rev Lett 2011;107(10):105506.
[18] Liang Z, Li J. Extreme acoustic metamaterial by coiling up space. Phys Rev Lett 2012;108(11):114301.

[19] Yang M, Ma G, Yang Z, Sheng P. Coupled membranes with doubly negative mass density and bulk modulus. Phys Rev Lett 2013;110(13):134301.

[20] Brunet T, Leng J, Mondain-Monval O. Soft acoustic metamaterials. Science 2013;342(6156):323-4.

[21] Xia B, Chen N, Xie L, Qin Y, Yu D. Temperature-controlled tunable acoustic metamaterial with active band gap and negative bulk modulus. Appl Acoust 2016;112:1-9.

[22] Liu XN, Hu GK, Sun CT, Huang GL. Wave propagation characterization and design of two-dimensional elastic chiral metacomposite. J Sound Vib 2011;330(11):2536-53.

[23] Zhu R, Liu XN, Hu GK, Sun CT, Huang GL. Negative refraction of elastic waves at the deep-subwavelength scale in a single-phase metamaterial. Nat Commun 2014;5(5):5510.

[24] Lakes R. Foam structures with a negative Poisson's ratio. Science 1987;235(4792):1038-40.

[25] Meng J, Deng Z, Zhang K, Xu X, Wen F. Band gap analysis of star-shaped honeycombs with varied Poisson's ratio. Smart Mater Struct 2015;24:095011.

[26] Grima JN, Gatt R, Alderson A, Evans KE. On the potential of connected stars as auxetic systems. Mol Simul 2005;31:925-35.

[27] Krushynska AO, Miniaci M, Kouznetsova VG, Geers MGD. Multilayered inclusions in locally resonant metamaterials: two-dimensional versus three-dimensional modeling. J Vib Acoust 2017;139(2):024501.

[28] Wen J, Zhao H, Lv L, Yuan B, Wang G, Wen X. Effects of locally resonant modes on underwater sound absorption in viscoelastic materials. J Acoust Soc Am 2011;130:1201-8.

[29] Veres IA, Berer T, Matsuda O. Complex band structures of two dimensional phononic crystals: analysis by the finite element method. J Appl Phys 2013;114(8):084701.

[30] Liu XN, Hu GK, Huang GL, Sun CT. An elastic metamaterial with simultaneously negative mass density and bulk modulus. Appl Phys Lett 2011;98:251907.

[31] Goffaux C, Sánchez DJ, Yeyati AL. Evidence of fano-like interference phenomena in locally resonant materials. Phys Rev Lett 2002;88(22):225502. 